Revue

de Sémantique

et Pragmatique

\section{Revue de Sémantique et Pragmatique}

41-42 | 2017

Cadrage sur la variation, le changement lexical et le changement grammatical en français actuel

\title{
Le marqueur LA en français actuel dans l'espace francophone : quelle description lexicographique pour quels usages?
}

Inka Wissner

\section{OpenEdition}

\section{Journals}

Édition électronique

URL : http://journals.openedition.org/rsp/463

DOI : $10.4000 /$ rsp.463

ISSN : $2610-4377$

Éditeur

Presses universitaires d'Orléans

Édition imprimée

Date de publication : 1 décembre 2017

Pagination : 79-97

ISSN : 1285-4093

Référence électronique

Inka Wissner, « Le marqueur LA en français actuel dans l'espace francophone : quelle description lexicographique pour quels usages ? », Revue de Sémantique et Pragmatique [En ligne], 41-42 | 2017, mis en ligne le 01 décembre 2018, consulté le 23 avril 2019. URL : http://journals.openedition.org/ rsp/463 ; DOI : 10.4000/rsp.463 


\section{LE MARQUEUR LÀ EN FRANÇAIS ACTUEL DANS L'ESPACE FRANCOPHONE : QUELLE DESCRIPTION LEXICOGRAPHIQUE POUR QUEL(s) USAGE(s)?}

Inka Wissner Université Paris-Sorbonne

1. INTRODUCTION 
abilité sociolinguistique avec les aires juxtaposées), ni, enfin, l'absence

associe trois plans : celui du signifié (aspects pragmasémantiques, relations paradigmatiques), celui du signifiant (morphologie, syn 
de réviser les regroupements dictionnairiques opérés, ni les défini que les travaux 
nçais ‘familier' (Rob, TLF). S'y ajoutent dans Antidote des emplois

e en lexicographie générale

mes. Pour LÀ, ces

emps son 
plus spécifiquement des articles basés sur ravaux affichent des différences disciplinaires,

e et les interjections y sont grosso modo analysés en tant qu'unités à valeur pragmatique et/ou discursive. S'y ajoutent des phénomènes qui affichent 
ire de France

lexi 
un $\ll \mathrm{L}$

is populaire à Abidjan semble enfin être seul à connaître

queur à Abidjan

s créoles (A). Enfin, la rela

ffet, les corpus textuels affichent, dans les aires francophones, une alement

rqueur discursif (absence de pause) (1993, 
ion), sans localisation ni source pour éviter d' orienter leur jugement ; ces exemples sont ici suivis d'un ' $\mathrm{T}$ ' (pour 'testé'). Voici les instructions, figurant après une phrase introductrice

e (« courant / rare / fam. / oral / autre : précisez »), la combina 
sur la reconnaissance des emplois courants, confirmant et

rmer

ynonymique dominante : [Abidjan] 
lobal que je désire transmettre; cette 
[Abidjan] faut filmer lui/qui vient

] (IFA)

Ex. [Abidjan] 
roduire, à laquelle je m'attarde avec une

de suite $(B, 28$ parle « d'ouverture »)

raconter' (cf. D à par.)

ur lequel je veux insister afin de t'amener à 
quatre cas de figure d'un point de vue variationnel, qui appellent différents trai

arquage du type 'oral surtout'

iorise le noyau du vocabulaire usuel de nos jours

gements formels, voire sémantiques, comme au Québec ( 
, permet d'obtenir des résultats tout relatifs qui ne sau

chent

yntaxique finale, 
Europe une restriction 
inence du concept

lusieurs aires comme LÀ, il s'agirait d'ajouter une entrée unique endre visibles l'expansion des éléments de la langue et leurs spécificités 
e et interaction verbale : un emploi de

os de "dialecto", "nivel" y "estilo de lengua" y el sentido propio de la dialectología » [1958],

deux cas de figure. 
on et contact de langues », in

um pragmatico-sémantique aux types prosodiques de

ce francophone à la

lumière de la base de données lexicographiques panfrancophone ",

liciers

d'A.D.G. », in

ières, processus de grammaticalisation », 
aphie de travail », Projet RELIEF/RLF, Nancy :

t marqué ? ", 